\title{
Wireless RF Distribution in Buildings using Heating and Ventilation Ducts
}

\author{
Christopher P. Diehl, Benjamin E. Henty, Nikhil Kanodia, and Daniel D. Stancil \\ Department of Electrical and Computer Engineering \\ Carnegie Mellon University, Pittsburgh, PA 15213
}

\begin{abstract}
An alternative method of distributing $R F$ in buildings is proposed in which the heating and ventilation ducts are used as waveguides. Because of the relatively low waveguide loss, this method may lead to more efficient RF distribution than possible with radiation through walls or the use of leaky coax. Further, the use of existing infrastructure could lead to a lower-cost system. Initial experimental results are presented that demonstrate duct-assisted propagation between nearby offices in a university building. An example method is described for obtaining efficient coupling between coax and 8" $x 12$ " rectangular duct over the 902-928 MHz ISM band.
\end{abstract}

\section{Introduction}

One of the challenges related to the installation of wireless networks in buildings is the need to predict RF propagation and coverage in the presence of complex combinations of shapes and materials in a building environment[1]. In general, the attenuation in buildings is higher than in free space, requiring more cells and higher power to obtain adequate coverage.

Over the past several years, an extensive wireless data network has been installed at Carnegie Mellon that provides coverage to about one half of the campus with raw speeds of two megabits per second[2-4]. With more than 100 access points, it is believed to be the largest local area network (LAN) installation anywhere. The effort required to provide a detailed description of the geometry and material composition of buildings on such a scale often causes system designers to resort to trial-and-error layouts.

An alternative to relying on direct propagation throughout a building is to install leaky coaxial cable[5]. Although this method lends itself to a systematic design procedure, the cost of the coax and its installation may be prohibitive. 
An alternative method of distributing RF in buildings is suggested by the recognition that every building is equipped with an RF waveguide distribution system-the HVAC ducts. The use of the HVAC ducts is also amenable to a systematic design procedure but should be significantly less expensive than other approaches since existing infrastructure is used and the RF is distributed more efficiently.

\section{Description of Proposed System}

We envision a distribution system in which RF is coupled into the ducts at a central location (perhaps near the air handling equipment) using inserted probes. These probes would be constructed much like existing coax-to-waveguide converters. In most installations, the ducts are largest near the central air handling equipment, and become smaller as they branch out to the various rooms. The branches and splits in the ducts would function as waveguide power splitters. Eventually, the RF would be radiated into rooms and offices through specially-designed louvers. Coverage in corridors or spaces shielded from louvers could be realized by placing passive reradiators in the sides of the ducts. As an alternative to special louvers to scatter the RF into local rooms, waveguide-to-coax coupling could be used to deliver the signal to wireless modems in the room.

The key idea underlying this distribution method is that low-loss electromagnetic waves can propagate in hollow metal pipes if the dimensions are sufficiently large compared to a wavelength. Since HVAC ducts are typically constructed of sheet metal, they are excellent waveguide candidates. The lowest frequency that can propagate in a given duct depends on the size and shape of the cross-section. For rectangular ducts, the cutoff frequency $f_{c o}$ for the lowest propagating mode is given by [6]

$$
f_{c o}=\frac{c}{2 a},
$$

where $c=3 \times 10^{8} \mathrm{~m} / \mathrm{s}$ is the velocity of light in free space, and $a$ is the largest dimension of the duct. For circular ducts, the lowest cutoff frequency is given by [6]

$$
f_{c o}=\frac{1.841 c}{2 \pi R},
$$


where $R$ is the radius of the duct. Minimum duct dimensions for several wireless bands are given in Table 1.

\begin{tabular}{|c|c|c|c|}
\hline Band (USA) & Lowest Frequency & $\begin{array}{c}\text { Minimum duct } \\
\text { width (Rectangular) }\end{array}$ & $\begin{array}{c}\text { Minimum duct } \\
\text { radius (Circular) }\end{array}$ \\
\hline Cellular & $824 \mathrm{MHz}$ & $18.2 \mathrm{~cm}(7.17 \mathrm{in})$ & $10.7 \mathrm{~cm}(4.20 \mathrm{in})$ \\
\hline ISM & $902 \mathrm{MHz}$ & $16.6 \mathrm{~cm}(6.55 \mathrm{in})$ & $9.75 \mathrm{~cm}(3.84 \mathrm{in})$ \\
\hline PCS & $1.85 \mathrm{GHz}$ & $8.1 \mathrm{~cm}(3.19 \mathrm{in})$ & $4.75 \mathrm{~cm}(1.87 \mathrm{in})$ \\
\hline ISM & $2.4 \mathrm{GHz}$ & $6.25 \mathrm{~cm}(2.46 \mathrm{in})$ & $3.66 \mathrm{~cm}(1.44 \mathrm{in})$ \\
\hline U-NII & $5.15 \mathrm{GHz}$ & $2.91 \mathrm{~cm}(1.15 \mathrm{in})$ & $1.71 \mathrm{~cm}(0.67 \mathrm{in})$ \\
\hline
\end{tabular}

Table 1. Minimum HVAC duct sizes supporting propagation in popular wireless bands.
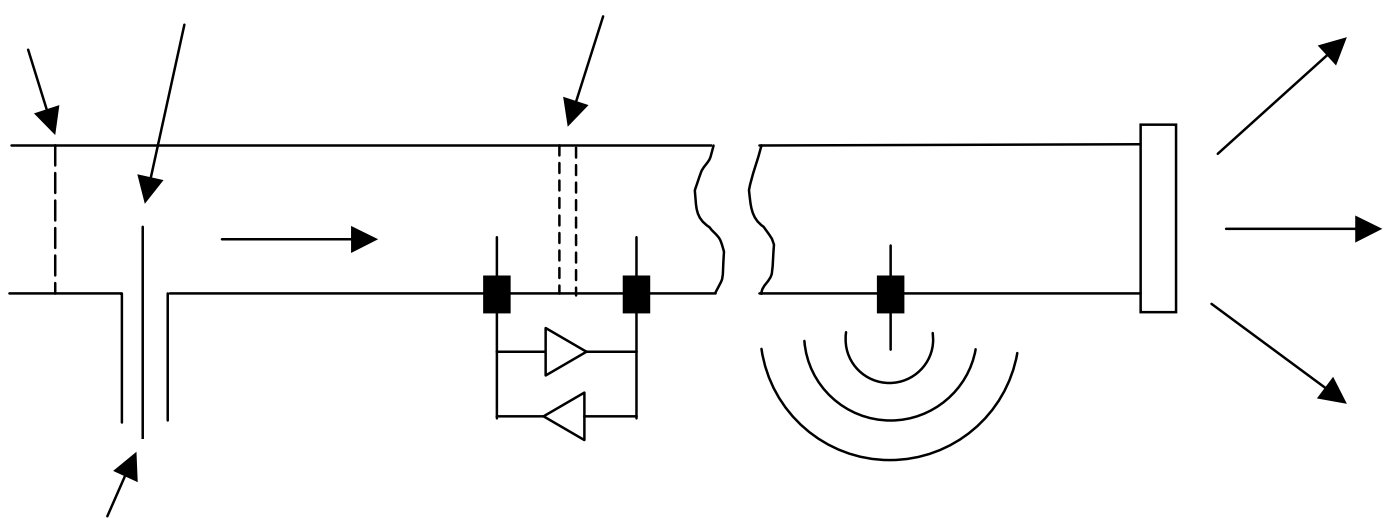

Figure 1. Major components of an HVAC RF distribution system.

The principal elements of an HVAC RF distribution system are shown schematically in Figure 1. One way to couple RF into the duct is by way of a probe antenna similar to those used in ordinary coax-to-waveguide adapters. An example coupler of this type is described in more detail in Section 4. A wire screen placed on one side of the probe could be used to accomplish 
impedance matching as well as unidirectional radiation. Such a screen would allow air to pass while reflecting RF energy.

Obstructions to RF such as cooling coils or fans may be occasionally encountered in the duct. In these cases probe-to-coax couplers could again be used to receive signals on one side of the obstruction and reradiate them on the other side. The couplers could be connected simply by low-loss coax or by bi-directional amplifiers if a boost in signal strength is needed. Coverage in corridors or spaces shielded from louvers could be realized by placing passive reradiators in the sides of the ducts. For example, a probe coupler could be connected to a small external monopole as shown in Fig. 1, or dielectric-filled slots could be cut in the side of the duct.

The various branches and splits in the ducts would function as waveguide power splitters. Depending on the geometry, it may be necessary to insert irises made of wire screens to ensure the desired power division at the branches. Dampers made of metal sheets to control the airflow would need to be replaced with insulating sheets (such as plastic) to minimize reflection of the RF. In addition, right-angle turns in the ducts may require the use of plastic rather than metal fins to guide both the air and the RF around the bend.

Eventually, the RF would be radiated into rooms and offices through the air vents. This would also require specially designed vents that disperse both the air and RF (commonly used vents made with metal louvers would block the RF). Alternatively, probe couplers could be used just inside the vent along with short segments of coax to deliver the signal locally to wireless modems.

\section{Concept Demonstration}

An initial experiment was performed to test the concept. Swept RF transmissions were made between two offices in one of the academic buildings on the CMU campus (Hamerschlag Hall). The measurement points were separated by about $6 \mathrm{~m}$ and there were two intervening walls. It was verified that the ducts into the two offices branch from a common trunk, thereby providing a waveguide path. The frequency was swept between $500 \mathrm{MHz}$ and $3 \mathrm{GHz}$ under two experimental conditions. In the first, simple dipole antennas tuned to $2.4 \mathrm{GHz}$ were placed in the offices about 
a meter away from the vents, but with the metal louvers in place. Figure 2 shows that the transmission loss between the two rooms was between 60 and $70 \mathrm{~dB}$ over this frequency range. In the second case, the dipoles were held against the front of the ducts with the metal louvers removed. The smallest duct diameter was $14.8 \mathrm{~cm}$ (5.8 in) leading to a lowest-order cutoff frequency of $1.19 \mathrm{GHz}$. As shown in Figure 3, the cutoff frequency is observed to be about 1.15 $\mathrm{GHz}$, in good agreement with theory. Above this cutoff, the signals are stronger by about $20 \mathrm{~dB}$ than in the direct propagation case. Note that no attempt was made to impedance-match or otherwise optimize the coupling into the duct.

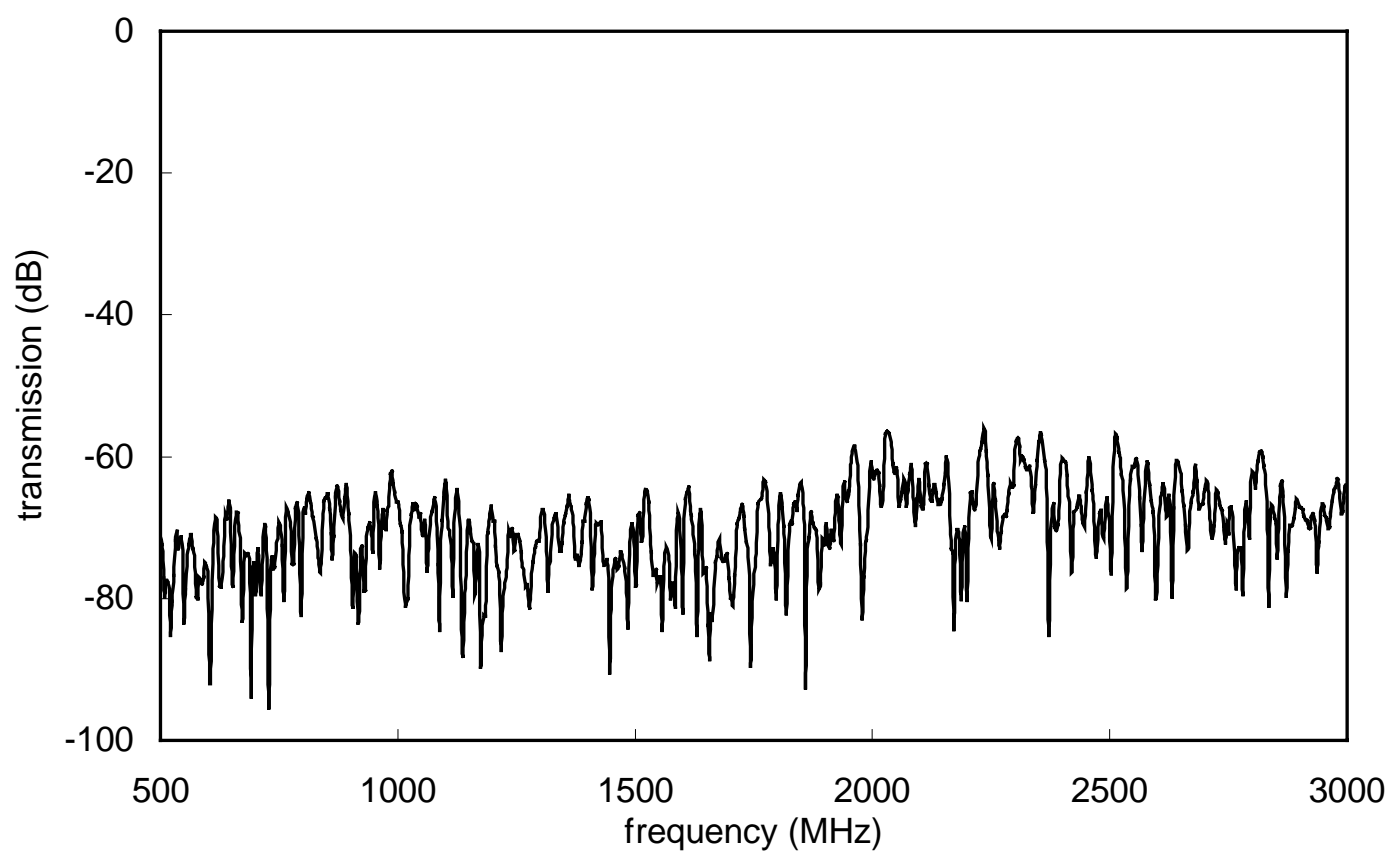

Figure 2. Transmission loss between two offices in Hamerschlag Hall without using ducts (metal louvers in place). 


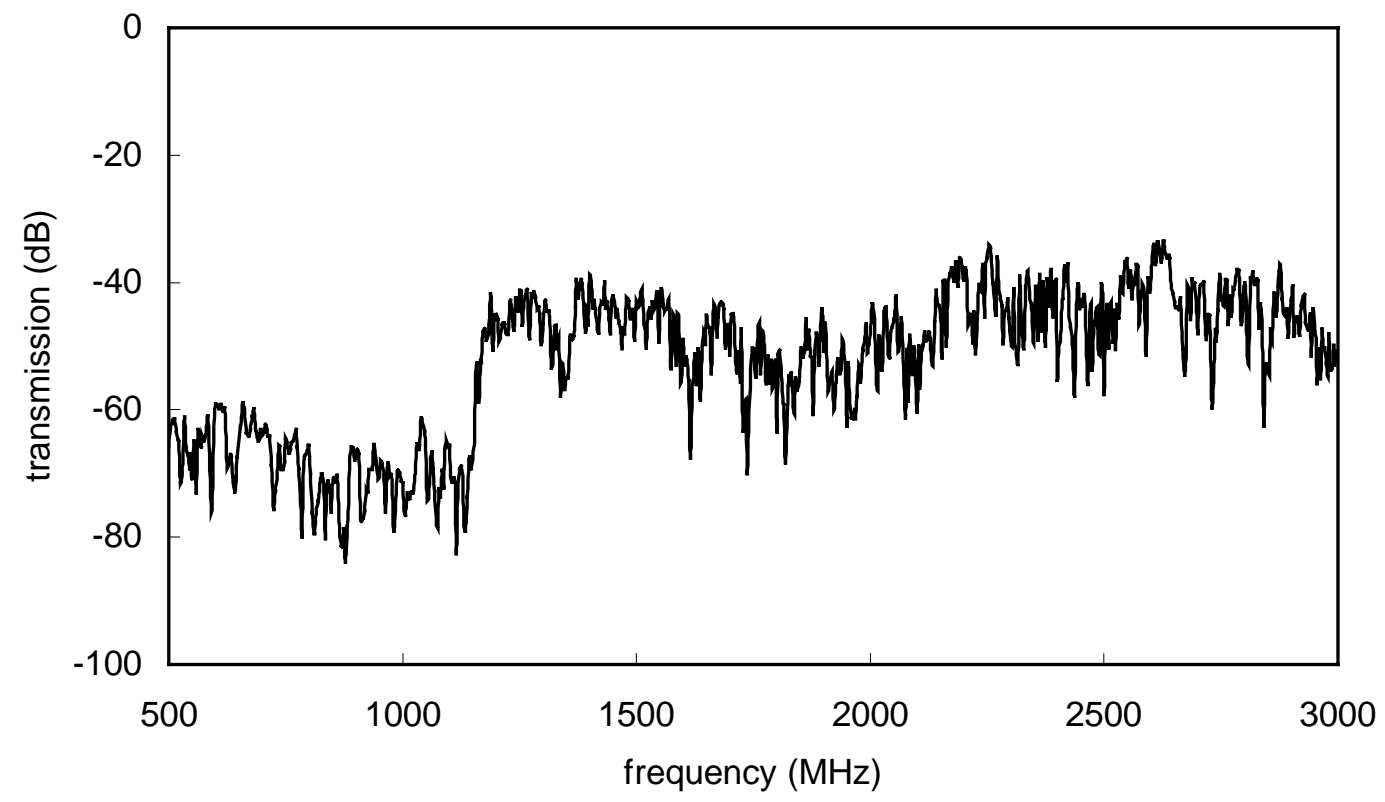

Figure 3. Transmission loss between two offices in Hamerschlag Hall using coupling through the HVAC ducts. The cutoff frequency of the duct is clearly seen.

\section{Example Coax-to-Duct Adapter for 915 MHz ISM Band}

Since the experiment described in Section 3 did not attempt to optimize the coupling into the duct, the design of an optimized probe coupler in an 8 " x 12 " rectangular duct was explored. The coupler was designed using a capped end-section of the duct, but it should be possible to use a wire grid as discussed earlier instead to allow airflow past the coupler. The design and dimensions of the coupler are shown in Figure 4. Using this design, a return loss in excess of 20 $\mathrm{dB}$ was obtained over the entire $915 \mathrm{MHz}$ ISM band (Figure 5). This means that more than $99 \%$ of the incident RF power is radiated into the duct, and demonstrates that efficient broadband coupling can be achieved. 


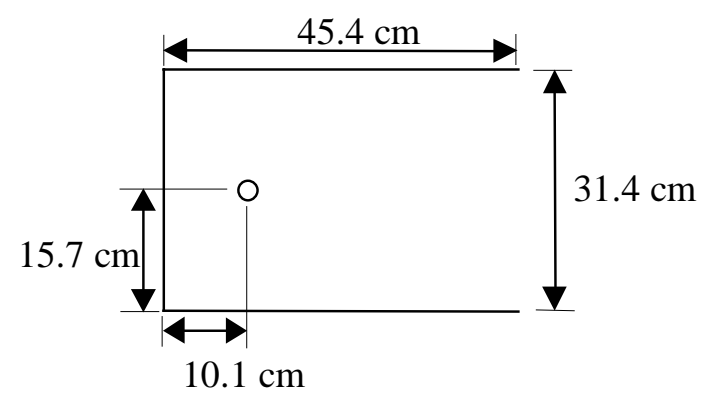

Top View

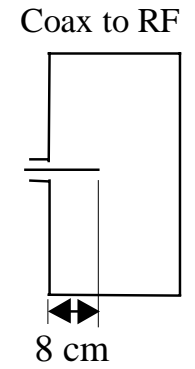

End View

Figure 4. Dimensions of the optimized probe coupler for the $915 \mathrm{MHz}$ ISM band.

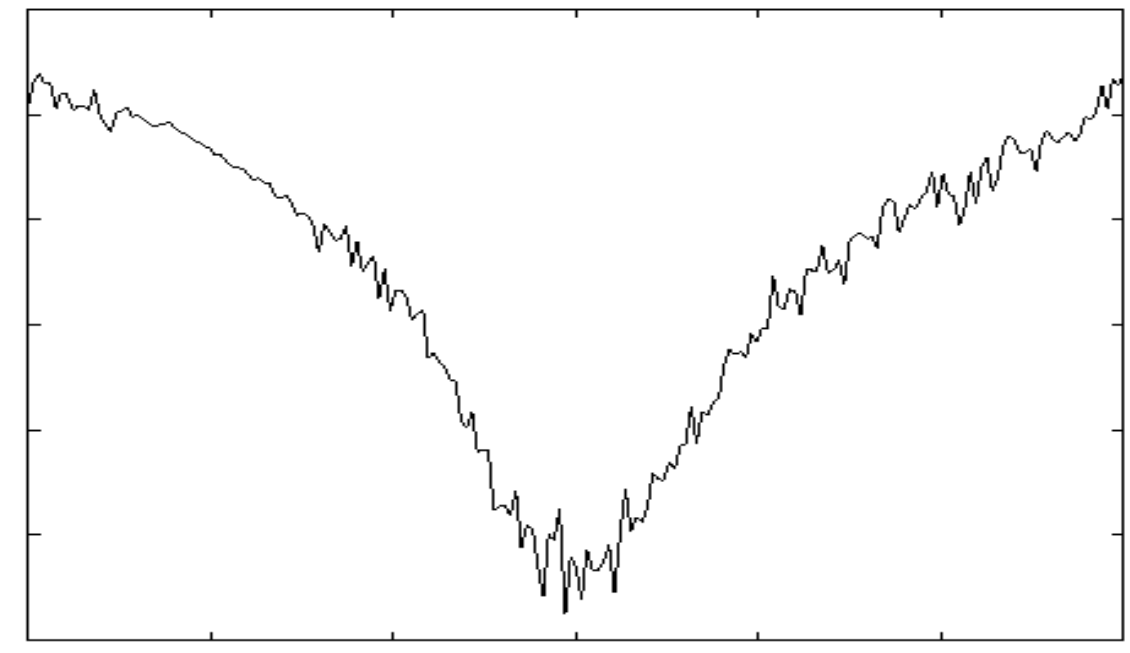

Figure 5. Return loss for the optimized coupler shown in Figure 4.

\section{Research Issues}

Although the preliminary experiments described in this paper support the feasibility of the HVAC RF distribution system, detailed research in a number of areas is needed to develop systematic design procedures. In the following we briefly comment on several of these. 


\section{Characterization of the RF channel}

Unlike conventional waveguide circuits, in most cases multiple waveguide modes will be above cutoff in the ducts. This multimode environment will lead to delay spread much like multipath in open propagation environments. Other sources of delay spread will be reflections from bends, junctions, and end-plates. It may be possible, for example, to minimize reflections from endplates with the use of foam absorbing material. In any event, delay spread and coherence bandwidth of such channels needs to be explored both theoretically and experimentally.

\section{Coupling into multimode ducts}

The existence of multiple propagating modes is a complication usually avoided in conventional waveguide circuits. Designs and design rules are needed for realizing efficient couplers in the various sizes and shapes of ducts that are commonly used, for each frequency band of interest.

\section{Mode conversion and cross-polarization in multimode ducts}

In the presence of multiple propagating modes, it is likely that the preferred strategy is to optimize coupling into the lowest-order, or dominant, waveguide mode. However, since HVAC ducts are not constructed with the same precision as actual waveguide circuits, mode conversion is likely at joints, seams, protrusions, and other imperfections. In addition to creating delay spread as discussed above, this mode conversion could lead to signal loss owing to excitation to orthogonally-polarized modes, as well.

\section{Power division at branches and tees}

To obtain satisfactory power distribution throughout a large building, it will be necessary to be able to determine and control the power division at branches and tees. This power division is also complicated by the existence of multiple propagating modes. The use of irises made using wire screens and grids should allow independent control of power division and airflow.

\section{Alternate construction of dampers and corner fins using dielectrics}

In existing HVAC systems, airflow is often controlled using adjustable metal dampers. Similarly, metal fins are often used to guide the air around sharp bends in the ducts. Both of these constructions potentially represent blockages for the RF. Designs should be explored using 
dielectric materials that allow the desired control of the air but which have minimal RF reflections.

\section{Coupling around obstructions}

As mentioned in Section 2, techniques are needed to couple around unavoidable obstructions in the ducts. Designs for both active and passive coupling need to be explored. The simplicity of passive probe couplers on either side of the obstruction connected by low-loss coax is attractive, but bi-directional amplifiers may be needed in some instances, as well. Such techniques could also be used to couple two otherwise unconnected duct systems.

\section{Design of louvers for dispersing both air and $R F$}

Common designs for louvers contain closely-spaced metal fins that would effectively block RF. Designs are needed that will disperse both the air and the RF into the room. For example, louvers made of dielectric materials could be made to minimize RF reflections while using conventional shapes to disperse the air. Going a step further, metal components could be embedded in the dielectric that are designed to scatter the RF uniformly into the space.

\section{Summary and Conclusions}

We have proposed an alternative technique for distributing RF communications signals in buildings using the HVAC ducts. Because existing infrastructure is used and the ducts exhibit losses that are low compared with direct propagation and leaky coax, such a system has the potential to be lower in cost and more efficient than either conventional method. Experimental results have been presented demonstrating duct-assisted propagation between offices in a university building, and efficient coax-to-duct coupling. Key research issues associated with developing practical systems have been briefly discussed.

\section{Acknowledgements}

We would like to acknowledge helpful discussions with B. Bennington and A. Hills regarding the design of large wireless LANs and the potential advantages of HVAC RF distribution 
systems. Appreciation is also expressed to D. Klein of McCarls Co. for information about the construction of HVAC systems and for providing duct samples for use in our experiments.

\section{References}

1. H. Hashemi, "The Indoor Radio Propagation Channel," Proceedings of the IEEE, Vol. 81, No. 7, pp. 943-968 (1993).

2. Alex Hills and David B. Johnson, "A Wireless Data Network Infrastructure at Carnegie Mellon University," IEEE Personal Communications, Vol. 3, No. 1, pp. $56-63$ (1996).

3. B. J. Bennington and C. R. Bartel, "Wireless Andrew: Experience Building a High-Speed Campus-wide Wireless Data Network," Proceedings of MobiCom'97, Budapest, Sept. 26, 1997.

4. Alex Hills, “Terrestrial Wireless Networks,” Scientific American, pp. 86-91, April 1998.

5. Dennis J. Burt, "In-Building Tricks: How to Design an In-Building Radio System," Communications, Vol. 31, No. 6, pp. 42, 44-47 (1994).

6. Robert E. Collin, Foundations for Microwave Engineering, $2^{\text {nd }}$ Edition, Chapter 3, McGraw Hill, 1992. 